\title{
TWO QUESTIONS OF L. VAŠ ON *-CLEAN RINGS
}

\author{
JIANLONG CHEN and JIAN CUI ${ }^{凶}$
}

(Received 8 December 2012; accepted 4 January 2013; first published online 8 March 2013)

\begin{abstract}
A *-ring $R$ is called (strongly) *-clean if every element of $R$ is the sum of a unit and a projection (that commute). Vaš ['*-Clean rings; some clean and almost clean Baer *-rings and von Neumann algebras', J. Algebra 324(12) (2010), 3388-3400] asked whether there exists a $*$-ring that is clean but not $*$-clean and whether a unit regular and $*$-regular ring is strongly $*$-clean. In this paper, we answer these two questions. We also give some characterisations related to $*$-regular rings.
\end{abstract}

2010 Mathematics subject classification: primary 16U99; secondary 16W10.

Keywords and phrases: *-clean ring, strongly *-clean ring, *-regular ring, strongly clean ring, unit regular ring.

\section{Introduction}

Rings in which every element is the product of a unit and an idempotent are said to be unit regular, and have been extensively studied. Camillo and Khurana [2] show that every element of a unit regular ring can also be written as the sum of a unit and an idempotent. Recall that an element of a ring $R$ is called clean if it is the sum of an idempotent and a unit, and $R$ is called clean if every element of $R$ is clean. Clean rings were introduced by Nicholson [4] in relation to exchange rings. In 1999, Nicholson [5] called an element of a ring $R$ strongly clean if it is the sum of a unit and an idempotent that commute with each other, and $R$ is strongly clean if each of its elements is strongly clean. Clearly, a strongly clean ring is clean, and the converse holds for abelian rings (that is, all idempotents in the ring are central). Local rings and strongly $\pi$-regular rings are well-known examples of strongly clean rings.

A ring $R$ is a $*$-ring (or ring with involution) if there exists an operation $*: R \rightarrow R$ such that for all $x, y \in R$,

$$
(x+y)^{*}=x^{*}+y^{*}, \quad(x y)^{*}=y^{*} x^{*} \quad \text { and } \quad\left(x^{*}\right)^{*}=x .
$$

This research was supported by the National Natural Science Foundation of China (10971024), the Specialized Research Fund for the Doctoral Program of Higher Education (20120092110020), the NSF of Jiangsu Province (BK2010393), and NSF of Anhui Educational Committee (KJ2010A126).

(C) 2013 Australian Mathematical Publishing Association Inc. 0004-9727/2013 \$16.00 
An element $p$ of a $*$-ring $R$ is said to be a projection if $p^{2}=p=p^{*}$. Recently, Vaš [6] introduced the concepts of a $*$-clean ring and a strongly $*$-clean ring. An element of a $*$-ring $R$ is called (strongly) $*$-clean if it can be expressed as the sum of a unit and a projection (that commute), and $R$ is called $*$-clean (respectively, strongly $*$-clean) if all of its elements are $*$-clean (respectively, strongly $*$-clean). Strongly $*$-clean rings are strongly clean and $*$-clean, and $*$-clean rings are clean, but Vaš asked whether there is a $*$-ring that is clean but not $*$-clean.

An involution $*$ of $R$ is called proper if $x^{*} x=0$ implies $x=0$ for any $x \in R$. Due to [1, Proposition 3], a $*$-ring $R$ is $*$-regular if one of the following equivalent conditions holds: (1) $R$ is a (von Neumann) regular and Rickart $*$-ring (that is, the right annihilator of each element is generated by a projection); (2) $R$ is regular and the involution is proper; (3) for every $x$ in $R$ there exists a projection $p$ such that $x R=p R$. It was shown that every $*$-abelian (that is, $*$-rings in which every projection is central) and $*$-regular ring is strongly $*$-clean [6]. Vaš asked whether a unit regular and $*$-regular ring is strongly $*$-clean.

In this paper, we answer the two questions raised by Vaš in [6] and investigate some properties of (strongly) *-clean rings. In particular, we show that a strongly clean ring $R$ is strongly $*$-clean if and only if the set of all projections of $R$ coincides with the set of all idempotents of $R$. In addition, we present some characterisations related to *-regular rings.

All rings considered in this paper are associative with unity. For a ring $R$, the set of all idempotents, all projections and all units of $R$ are denoted by $\operatorname{Id}(R), P(R)$ and $U(R)$, respectively. The symbol $l(X)$ (respectively, $r(X)$ ) stands for the left (respectively, right) annihilator of a subset $X \subseteq R$. We write $M_{n}(R)$ for the ring of all $n \times n$ matrices over $R$.

\section{Main results}

We begin with the following result.

THeOREM 2.1. Let $R$ be $a *$-ring and $p \in P(R)$. Then $a \in p R p$ is strongly $*$-clean in $R$ if and only if a is strongly $*$-clean in $p R p$.

Proof. Assume that $a$ is strongly $*$-clean in $p R p$. Then there exist $e \in P(p R p)$ and $u \in U(p R p)$ such that $a=e+u$ and $u e=e u$. Let $f=e+(1-p)$ and $v=u-(1-p)$. Then $a=f+v$ and $f v=v f$, where $f \in P(R)$ and $v \in U(R)$. So $a$ is strongly $*$-clean in $R$.

Conversely, suppose that $a \in p R p$ is strongly $*$-clean in $R$. Let $a=e+u$ with $e \in P(R), u \in U(R)$ and $u e=e u$. Since $a=$ pap, $1-p \in r(a) \cap l(a)$. By [5, Theorem 2], $r(a) \subseteq e R$ and $l(a) \subseteq R e$. So $1-p \in e R \cap R e=e R e$, and then $(1-p) e=e(1-p)$, whence $e p=p e$. Note that both $e$ and $p$ are projections. Then $p e p \in P(p R p)$. Since $a p=p a$ and $u=a-e$, we obtain $u p=p u$. It follows that $p u p \in U(p R p)$, and pep commutes with $p u p$. Therefore, $a=p e p+p u p$ is strongly $*$-clean in $p R p$. 
COROLlaRy 2.2. If $R$ is a strongly $*$-clean ring, then $p R p$ is strongly $*$-clean for any $p \in P(R)$.

The following result is crucial for constructing a counterexample of a $*$-ring that is strongly clean but not strongly $*$-clean.

Theorem 2.3. Let $R$ be a $*$-ring. Then $R$ is strongly $*$-clean if and only if $R$ is strongly clean and $P(R)=\operatorname{Id}(R)$.

Proof. Suppose that $R$ is strongly $*$-clean. We only need to show that $\operatorname{Id}(R) \subseteq P(R)$. For any $e^{2}=e \in R$, we have $e=p+u$ where $p \in P(R), u \in U(R)$ and $e, p$ and $u$ commute with each other. If $p=0$ then $e=1$, and if $p=1$ then $e=0$. So we may assume that $p \neq 0$ and $p \neq 1$. Then $p R p$ and $(1-p) R(1-p)$ are nonzero $*$-rings. Now, multiplying $e=p+u$ by $p$ yields $e p=p+u p$. It follows that $-u p=p-e p=$ $(1-e) p \in U(p R p) \cap \operatorname{Id}(p R p)=\{p\}$. Thus ep $=0$. Analogously, multiplying both sides of $e=p+u$ by $1-p$ gives $e(1-p)=u(1-p) \in U((1-p) R(1-p)) \cap$ $\operatorname{Id}((1-p) R(1-p))=(1-p)$. So $e-e p=1-p$. Since $e p=0, e=1-p \in P(R)$. This proves that $\operatorname{Id}(R)=P(R)$. The other direction is trivial.

According to [6], if $R$ is a *-ring, $M_{n}(R)$ has a natural involution inherited from $R$ : if $A=\left(a_{i j}\right) \in M_{n}(R), A^{*}$ is the transpose of $\left(a_{i j}^{*}\right)$. Henceforth we consider $M_{n}(R)$ as a *-ring with respect to this natural involution. Vaš [6, Proposition 4] showed that $M_{n}(R)$ is a $*$-clean ring whenever $R$ is $*$-clean. Since, for $n \geq 2, M_{n}(R)$ has idempotents that are not projections, Theorem 2.3 implies the following result.

Corollary 2.4. Let $R$ be a $*$-ring. Then $M_{n}(R)$ is not strongly $*$-clean for any $n \geq 2$.

Note that a local ring $R$ with any involution $*$ is strongly $*$-clean. So, $M_{n}(R)$ is $*$-clean, but it is not strongly $*$-clean if $n \geq 2$. Vaš [6] asked whether there is a $*$-ring that is clean but not $*$-clean. We answer this question affirmatively by the following example.

ExAmple 2.5. Let $R=\mathbb{Z}_{2} \oplus \mathbb{Z}_{2}$, where $\mathbb{Z}_{2}$ is the ring of integers $\mathbb{Z}$ modulo 2. Then $R$ is strongly clean and $R=\operatorname{Id}(R)$. Define a map $*: R \rightarrow R$ by $(a, b)^{*}=(b, a)$. Then $*$ is an involution of $R$. Note that $P(R)=\{(0,0),(1,1)\} \neq \operatorname{Id}(R)$. By Theorem $2.3, R$ is not strongly $*$-clean, and thus not $*$-clean because $R$ is commutative.

REMARK 2.6. Example 2.5 shows that strongly clean $*$-rings need not be $*$-clean. The following implications hold:

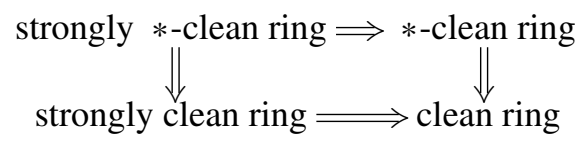

In this diagram, each of the implications is irreversible, and there are no other implications between these rings. 
Recall that a ring $R$ is right $P$-injective if $\operatorname{lr}(a)=R a$ for each $a \in R$. Regular rings are clearly right P-injective.

Proposition 2.7. Let $R$ be $a *$-ring. Then the following are equivalent.

(1) $R$ is regular and the involution is proper (that is, $R$ is *-regular).

(2) $R$ is right $P$-injective and the involution is proper.

(3) For every $a \in R, R a=R a^{*} a$.

Proof. $(1) \Rightarrow(2)$. This is clear.

$(2) \Rightarrow(3)$. Given $a \in R$, let $y \in r\left(a^{*} a\right)$. Then $a^{*} a y=0$. It follows that $0=y^{*} a^{*} a y=$ $(a y)^{*}(a y)$. Since the involution $*$ is proper, $a y=0$. Thus $y \in r(a)$, which implies that $r\left(a^{*} a\right)=r(a)$. By the right P-injectivity of $R$, we obtain $\operatorname{Ra}=\operatorname{lr}(a)=\operatorname{lr}\left(a^{*} a\right)=$ $R a^{*} a$.

(3) $\Rightarrow(1)$. For any $a \in R$, there exists $t \in R$ such that $a=t a^{*} a$. Then $a t^{*} a=$ $\left(t a^{*} a\right) t^{*} a=t\left(a^{*} a t^{*}\right) a=t\left(t a^{*} a\right)^{*} a=t a^{*} a=a$. Thus $R$ is a regular ring. To show that the involution is proper, we let $x^{*} x=0$ with $x \in R$. Then $R x=R x^{*} x=0$, so $x=0$, as desired.

A ring $R$ is strongly regular if it is an abelian regular ring, or equivalently, for any $a \in R, a=e u=u e$ for some $e \in \operatorname{Id}(R)$ and $u \in U(R)$ (see [5]). It is well known that strongly regular rings are unit regular, and unit regular rings are regular.

Proposition 2.8. Let $R$ be $a *$-ring. Then the following are equivalent.

(1) $R$ is strongly regular and the involution is proper.

(2) $R$ is strongly regular and $P(R)=\operatorname{Id}(R)$.

(3) $R$ is *-abelian and, for every $a \in R$, there exist $p \in P(R)$ and $u \in U(R)$ such that $a=p+u$ and $a R \cap p R=0$.

(4) For every $a \in R, a=p u=u p$ for some $p \in P(R)$ and $u \in U(R)$.

Proof. (1) $\Rightarrow(2)$. In view of Proposition 2.7, $R$ is *-regular. By [6, Lemma 3], $P(R)=\operatorname{Id}(R)$ since $R$ is abelian.

$(2) \Rightarrow(3)$. Note that every abelian $*$-ring is $*$-abelian. So the rest follows from [2, Theorem 1].

(3) $\Rightarrow(4)$. Let $a \in R$. Then there exist $1-p \in P(R)$ and $u \in U(R)$ such that $a=$ $(1-p)+u$ and $a R \cap(1-p) R=0$. Since $R$ is $*$-abelian, $a(1-p) \in a R \cap R(1-p)=$ $a R \cap(1-p) R=0$. Then $a=a p$. Note that $a=(1-p)+u$. Hence, $a=p u=u p$.

$(4) \Rightarrow(1)$. It suffices to show that the involution is proper. Let $x \in R$ with $x^{*} x=0$. Then $x=p u=u p$ for some $p \in P(R)$ and $u \in U(R)$. So we have $0=x^{*} x=(p u)^{*} p u=$ $u^{*} p u$. Notice that $U(R)^{*}=U(R)$. Thus $p=0$, and so $x=0$. This proves that the involution $*$ of $R$ is proper.

A ring $R$ is said to have stable range 1 provided that whenever $a R+b R=R$ for any $a, b \in R$, there exists $t \in R$ such that $a+b t \in U(R)$. Next we give some characterisations of unit regular and $*$-regular rings. 
Theorem 2.9. Let $R$ be $a *$-ring. Then the following are equivalent.

(1) $R$ is unit regular and the involution is proper.

(2) $R$ is unit regular and $*$-regular.

(3) For every $a \in R, a=p u$ where $p \in P(R)$ and $u \in U(R)$.

(4) For every $a \in R, a=v q$ where $q \in P(R)$ and $v \in U(R)$.

Proof. (1) $\Rightarrow(2)$. This follows by Proposition 2.7 .

$(2) \Rightarrow(3)$. For any $a \in R$, there exist $e \in \operatorname{Id}(R)$ and $w \in U(R)$ such that $a=e w$. Since $R$ is $*$-regular, $e R=p R$ for some projection $p \in R$. Thus $e=p e$ and $e R+(1-p) R=R$. Since $R$ is unit regular, by [3, Proposition 4.12], $R$ has stable range 1. So there exists $t \in R$ satisfying $e+(1-p) t \in U(R)$. Let $v=e+(1-p) t$. Then $p e=p v$. It follows that $e=p e=p v$, and $a=e w=p(v w)$. Write $u=v w$. Then $a=p u$ and $u \in U(R)$.

(3) $\Rightarrow(4)$. Given $a \in R$, let $b=a^{*}$. By hypothesis, $b=p u$ with $p \in P(R)$ and $u \in$ $U(R)$. Then $a=b^{*}=u^{*} p$. Write $v=u^{*}$ and $q=p$. Then $v \in U(R), q \in P(R)$ and $a=v q$.

$(4) \Rightarrow(1)$. The ring $R$ is clearly unit regular, so we only need to show that the involution is proper. Let $a \in R$ with $a^{*} a=0$. By (4), $a^{*}=v q$ for some $v \in U(R)$ and $q \in P(R)$. Thus $0=a^{*} a=(v q)\left(q v^{*}\right)=v q v^{*}$. So $q=0$, which implies that $a=0$, as required.

Definition 2.10. A $*$-ring $R$ is called $*$-unit regular if $R$ satisfies the conditions in Theorem 2.9.

Proposition 2.11. Let $R$ be $a *$-ring and $n$ a positive integer. The following are equivalent.

(1) $M_{n}(R)$ is *-unit regular.

(2) $R$ is unit regular and $a_{1}^{*} a_{1}+a_{2}^{*} a_{2}+\cdots+a_{n}^{*} a_{n}=0$ implies $a_{i}=0$ for all $i$.

Proof. $(1) \Rightarrow(2)$. Since $M_{n}(R)$ is $*$-unit regular, it is unit regular. By [3, Corollary 4.7], $R$ is unit regular. Suppose that $a_{1}^{*} a_{1}+a_{2}^{*} a_{2}+\cdots+a_{n}^{*} a_{n}=0$ for some $a_{i} \in R$. Let

$$
A=\left(\begin{array}{cccc}
a_{1} & 0 & \cdots & 0 \\
a_{2} & 0 & \cdots & 0 \\
\vdots & \vdots & \ddots & \vdots \\
a_{n} & 0 & \cdots & 0
\end{array}\right) \in M_{n}(R) .
$$

Then $A^{*} A=0$. Since the involution $*$ of $M_{n}(R)$ is proper, $A=0$. Thus, $a_{1}=a_{2}=\cdots=$ $a_{n}=0$.

(2) $\Rightarrow(1)$. By [3, Corollary 4.7], $M_{n}(R)$ is unit regular since $R$ is a unit regular ring. Next we show that the involution $*$ of $M_{n}(R)$ is proper. Let $A=\left(a_{i j}\right) \in M_{n}(R)$ with $A^{*} A=0$. Then

$$
a_{1 j}^{*} a_{1 j}+a_{2 j}^{*} a_{2 j}+\cdots+a_{n j}^{*} a_{n j}=0
$$

where $j=1, \ldots, n$. By hypothesis, $a_{i j}=0$ for all $i, j$. Thus $A=0$, and the proof is complete. 
Proposition 2.11 yields the following examples.

Example 2.12. Clearly, the number fields $\mathbb{R}$ and $\mathbb{C}$ are unit regular rings.

(1) Let $*=1_{\mathbb{R}}$ be the identity map of $\mathbb{R}$. Then $M_{n}(\mathbb{R})$ is $*$-unit regular.

(2) Define an involution $*$ of $\mathbb{C}$ by $x \mapsto \bar{x}$, where $\bar{x}$ is the conjugation of $x$. By a direct computation, $M_{n}(\mathbb{C})$ is $*$-unit regular.

(3) Let $R=\mathbb{R} \times \mathbb{R}$ be a ring with the usual addition and multiplication. Let $*=1_{R}$. Then $R$ is unit regular and $M_{n}(R)$ is *-unit regular.

(4) Let $*: x \mapsto x$ be an involution of $\mathbb{Z}_{2}$. By Proposition $2.11, M_{2}\left(\mathbb{Z}_{2}\right)$ is not $*$-unit regular because $1^{*} \cdot 1+1^{*} \cdot 1=0$ but $1 \neq 0$.

In [5], Nicholson asked whether a unit regular ring is strongly clean; this is still an open problem. Vaš [6] raised the question of their *-versions. We give a negative answer.

Example 2.13. Let $R$ be a $*$-ring as given in Example 2.12(1), (2) or (3). Then $M_{2}(R)$ is $*$-unit regular. But $M_{2}(R)$ is not strongly $*$-clean by Corollary 2.4.

According to Example 2.12(4), one may see that the matrix ring of a $*$-unit regular ring need not be $*$-unit regular. However, we have the following result for the corner rings.

Proposition 2.14. If $R$ is a $*$-unit regular ring, then $p R p$ is $*$-unit regular for every $p \in P(R)$.

Proof. Let $p \in P(R)$ and $S=p R p$. In view of [3, Corollary 4.7], $S$ is unit regular since $R$ is unit regular. Let $a \in S(\subseteq R)$ with $a^{*} a=0$. Since $R$ is $*$-unit regular, we get $a=0$. So the involution of $S$ is proper. By Theorem 2.9, $S=p R p$ is $*$-unit regular.

\section{Acknowledgement}

The authors would like to thank the referee for valuable comments.

\section{References}

[1] S. K. Berberian, Baer $*$-Rings, Grundlehren der Mathematischen Wissenschaften, 195 (Springer, Berlin, 1972).

[2] V. P. Camillo and D. Khurana, 'A characterization of unit regular rings', Comm. Algebra 29(5) (2001), 2293-2295.

[3] K. R. Goodearl, Von Neumann Regular Rings, Monographs and Studies in Mathematics, 4 (Pitman, London, 1979).

[4] W. K. Nicholson, 'Lifting idempotents and exchange rings', Trans. Amer. Math. Soc. 229 (1977), 269-278.

[5] W. K. Nicholson, 'Strongly clean rings and Fitting's lemma', Comm. Algebra 27(8) (1999), 35833592.

[6] L. Vaš, '*-Clean rings; some clean and almost clean Baer *-rings and von Neumann algebras', J. Algebra 324(12) (2010), 3388-3400. 
JIANLONG CHEN, Department of Mathematics, Southeast University, Nanjing 210096, PR China

e-mail: jlchen@ seu.edu.cn

JIAN CUI, Department of Mathematics, Anhui Normal University, Wuhu 241000, PR China

e-mail: jcui2006@126.com, jcui1635@gmail.com 